Reliability of Moral Decision Making: Evidence from the Trolley Dilemma Corentin J. Gosling ${ }^{1,2,3 *}$ \& Bastien Trémolière ${ }^{2 *}$

Manuscript (registered report) accepted for publication in QJEP

Author Note

Corentin J. Gosling

${ }^{1}$ DysCo Lab, Paris Nanterre University, France

${ }^{2}$ EA7352, Nimes University, France

${ }^{3}$ Université de Paris, Laboratoire de Psychopathologie et Processus de Santé, F-92100

Boulogne Billancourt, France.

Bastien Trémolière

${ }^{2}$ EA7352, Nimes University

Correspondence concerning this article should be addressed to Corentin J. Gosling, 200 Avenue de la République, Paris Nanterre University, 92000, Nanterre, France (Email: corentin.gosling@parisnanterre.fr)

*Both authors contributed equally to this manuscript. 


\begin{abstract}
The application of framing effects in the field of moral judgment has offered a golden opportunity to assess the reliability of people's moral judgments and decisions. To date, however, these studies are still scarce and they suffer from multiple methodological issues. Therefore, the present study aims to provide further insights into the reliability of moral judgments while fixing these methodological shortcomings. In the current study, we employed the classic trolley dilemma moral decision-making paradigm to determine the extent to which moral decisions are susceptible to framing effects. A total of 1040 participants were included in the study. The data revealed that choices of participants did not significantly differ between the two frames. Equivalence tests confirmed that the associated effect size was very small. Further exploratory analyses revealed an unplanned interaction between the framing effect and the target of the framing manipulation. This result became from marginally statistically significant to insignificant following different sensitivity analyses. The implications and limitations of these findings and directions for future research are discussed.
\end{abstract}

Keywords: Morality; Decision-making; Framing effect; Bias; Reliability 
Reliability of Moral Decision Making: Evidence from the Trolley Dilemma

In the past 50 years, the exploration of the psychological processes underlying our moral judgments has led to countless studies. In this time window, several theoretical models have followed one after another. Moral judgment has first been assumed to be underlined by reasoning processes (Kohlberg, 1969) only to be later recast as an intuitive activity, in which reasoning is only triggered at a second stage to justify one's initial intuition (Haidt, 2001). Since then, some authors have argued that both intuitive/emotional and deliberated components are at game at the time of making moral judgments (Greene et al., 2008; Greene et al., 2004; Greene et al., 2001). Along with a greater knowledge and understanding of the psychological processes underlying moral decision-making, some authors have pointed out multiple concerns regarding the reliability of moral decision-making (e.g., Sinnot-Armstrong, 2008). Because consensus on the reliability of moral decision-making has not yet been reached, this study aims to provide further insights into the reliability of judgments in moral dilemmas.

\section{Framing effects in decision making}

In an attempt to question the reliability of moral decision-making, some authors have explored the influence of the framing effects on moral decisions. This cognitive bias commonly refers to how people's choices are shaped by the way they are formulated (Tversky \& Kahneman, 1981). One of the most striking examples of framing effects is known as the Asian-disease problem (Tversky \& Kahneman, 1981). In this problem, participants are told that 600 people suffer from a fatal disease. To combat this disease, participants must choose between a treatment with a sure outcome of saving some of the afflicted people and a second treatment with a risky outcome of either saving or killing all afflicted people. Although the two options have the same expected value, people are typically risk-averse in positively-framed scenarios (lives saved) and risk- 
seeking in negatively-framed scenarios (lives lost; Kühberger et al., 1999). Multiple variants of the framing effects have been explored, which have been classified into three categories based upon their structure: the risky choice framing effect, the attribute framing effect and the goal framing effect (Levin et al., 1998).

The basic principle of a framing problem consists in the manipulation of the formulation of key features of the problem. In the risky choice framing effect, framing is manipulated through the formulation of two independent options which only differ in the certainty in their outcome (one outcome being sure, the other being risky; e.g., see the Asian-disease problem presented above). In the attribute framing effect, the framing manipulation targets the formulation of one attribute of a given problem. A classic example of attribute framing requires participants to judge the taste of a positively-framed meat (i.e., described as $85 \%$ lean) or of a negatively-framed meat (i.e., described a 15\% fat; Levin \& Gaeth, 1988). Results showed that participants judged meat as better tasting when it was described in a positive versus negative frame, even though the two descriptions convey identical information. In the goal framing effect, finally, the framing manipulation targets the formulation of the relationship between a behavior and its consequence. The relationship itself is the subject of the framing manipulation. For example, Meyerowitz and Chaiken (1987) showed that telling participants that 'not performing breast self-examination decreased chances of detecting a tumor at a treatable stage' strengthens their propensity to perform a breast exam, compared to telling them that 'performing a breast self-examination increased chances of detecting a tumor at a treatable stage'.

Among the multiple attempts to understand the psychological processes underlying these framing effects, researchers have largely relied on the dual-process theories of thinking (De Martino et al., 2006; Kahneman, 2011; Bilandzic et al., 2017; Murch \& Krawczyk, 2013). 
Although there are many dual-process models (Evans, 2008; Evans \& Over, 1996; Epstein, 1994; Kahneman 2011; Sloman, 1996; Stanovich, 1999), the generic version posits that people have two distinct thinking systems: the intuitive system, which is fast, and cognitively effortless, and the analytic system, which is slow, and greedier in cognitive resources. According to this theory, framing susceptibility captures the activation of the intuitive system, which is largely sensitive to superficial information (Kahneman \& Frederick, 2007). That is, people staying at the intuitive system level would largely be sensitive to the formulation, leading in turn to differences in the acceptance or rejection of a given option.

\section{Reliability and framing in moral dilemmas}

The framing paradigm appears as a golden opportunity to investigate the reliability of moral decision-making, as simple variations in problem formulation do not affect the content of the problem. Therefore, unlike some other operationalizations used to assess reliability of moral decision-making (such as the modification of some contextual elements of the decision problems; Demaree-Cotton, 2016; Helzer et al., 2017), framing susceptibility can serve as a proper measure of the reliability of moral decisions. The advantages are twofold: This procedure makes it possible to present the participants with the same problem (i.e., with an exactly similar context or consequences) and to assess the reliability of moral decision-making by exploring the influence of irrelevant and superficial cues, such as wording. Because a careful examination of the alternatives should decrease reliance on the superficial formulation and should thus decrease framing susceptibility (e.g., Seta et al., 2017), larger framing effects reflect lower reliability (e.g., Sinnott-Armstrong, 2011). 
Historically, moral decision-making has taken a great interest in studying sacrificial moral dilemmas (see Christensen \& Gomila, 2012), although one has to note that the study of moral judgment captures a wider range of situations (for instance, decisions about blame, guilt, or punishment, among others). The basic structure of a moral dilemma features a fictitious situation in which one has to decide between actively killing someone to save a greater number of persons, as exemplified in the famous trolley dilemma (Foot, 1978, Thomson, 1985). Among the many dilemmas designed, the bystander and the footbridge dilemma stand as flagship variations of the trolley dilemma.

The bystander version of the trolley dilemma depicts a situation in which participants are told to envision themselves standing near a railroad lever. A runaway trolley barreling down the track will kill five people working on the track. To prevent the accident, participants are told that pulling the lever will divert the trolley to a secondary track which will only kill one worker standing on this secondary track, instead of the five workers on the main track. In the footbridge version, participants are told to envision themselves standing beside another person on a footbridge that spans a railroad track. A runaway trolley is barreling down the railroad track toward five people working on it and who will be killed in the accident. To prevent the accident, participants are told that pushing the person standing next to them off the footbridge onto the trolley track will kill that person but will slow the trolley enough to save the five workers. These two scenarios are constructed in that both actions (pushing a lever in the bystander scenario or pushing a man in the footbridge scenario) produce the same consequence (killing 1 person and saving 5 people). Participants typically make utilitarian judgments and are willing to pull the lever in the bystander scenario but they typically make deontological judgments and are resistant to pushing the man in the footbridge scenario (Greene et al., 2001; 2004). 
If the framing effects have been widely studied in the field of moral decision making ${ }^{1}$, to our knowledge, only four studies have assessed their role in standard trolley-like dilemmas ${ }^{2}$, with the potential to afford cues about reliability of people's moral decisions (Broeders et al., 2011; Pastötter et al., 2013; Cao et al., 2017; Petrinovich \& O’Neill, 1996). Results, however, are hardly conclusive. Petrinovich \& O’Neill (1996) found that participants were susceptible to the framing effect in the bystander scenario, and Pastötter et al. (2013) showed that participants resisted the framing effect in the footbridge scenario. In line with these results, Cao et al. (2017) confirmed that participants resisted framing manipulation in the 'footbridge scenario' while they were susceptible to the framing manipulation in the 'bystander scenario'. On the opposite, one study showed that participants resisted the framing manipulation in the 'bystander scenario' while they were susceptible to the framing manipulation in the 'footbridge scenario' (Broeders et al., 2011), which runs counter the aforementioned findings.

Taken together, these mixed results make it difficult to reach a clear conclusion regarding the reliability of moral judgments in moral dilemmas. However, there are important reasons to believe that the differences in the results observed lie in the heterogeneity in the designs employed as well as the presence of methodological issues. More precisely, Broeders et al. (2011) have explored the framing effect in the trolley dilemma by explicitly (using a priming

\footnotetext{
${ }^{1}$ For instance, the famous Asian disease problem has also been used to assess moral beliefs (for instance, see Sinnott-Armstrong, 2008).

${ }^{2}$ It is worth noting that many more studies have been conducted which manipulated 'framing' in the context of trolley-like dilemmas (for a review, see Demaree-Cotton, 2016). However, many of these studies relied on manipulations of presentation order or context of scenarios, which is much further away of the initial description of the framing effect by Tversky and Kahneman (1981). In the present research, we intentionally use Tversky and Kahneman's definition of the framing effect as a manipulation of wording and we discuss only the research that closely rely on this paradigm.
} 
scenario) or implicitly (using a subliminal paradigm) priming the rule 'Save lives' or 'Do not kill' prior to participants answer the moral dilemma. Pastötter et al. (2013) assessed framing susceptibility in the footbridge scenario by varying the formulation of the question asked to the participants. In their study, one group of participants was presented with an active question ('Do you think it is appropriate to be active and push the man?') while a second group was presented with a passive question ('Do you think it is appropriate to be passive and not push the man?'). Experimentally speaking, making vary the formulation of a prime (as it was the case in Broeders et al., 2011) or a question (as it was the case in Pastötter et al., 2013) looks like attribute framing at first glance, since an attribute is the subject to the framing manipulation. However, this manipulation cannot be described as pure case of attribute framing since the formulation of the problem remains unchanged (Levin et al., 1998). Cao et al. (2017) and Petrinovich \& O’Neill (1996) explored framing susceptibility in the trolley dilemma by presenting participants with either the positive (lives saved) or negative consequences (lives lost) of the two possible actions (pushing the man/lever or doing nothing). In other words, participants faced either a positivelyframed problem ('If you perform the action, 5 people will survive and if you do nothing, one person will survive') or a negatively-framed problem ('If you perform the action, one person will die and if you do nothing, 5 people will die'). Again, although the manipulations of these two studies are close to goal framing (i.e., the relationship between a behavior and a relationship are the target of the framing manipulation), they cannot be described as pure cases of goal framing since the two behaviors (performing an action and doing nothing) and their consequences were presented simultaneously. Because of these concerns in the method used, we believe that further research using more refined methods are needed to afford more reliable conclusions.

\section{The current study}


Our study first aims to address the issues reported, clarifying whether moral decision-making is affected by the goal framing effect in trolley-like dilemmas. We will exclusively address the goal framing effect, because it appears to be the only form of framing effects which can be studied in trolley-like dilemmas without requiring too many changes in the initial material. In line with previous studies, we hypothesize that participants will be susceptible to the goal framing effect by accepting sacrificial harm more in the positive compared to the negative frame (Broeders et al., 2011; Cao et al., 2017; Petrinovich \& O’Neill, 1996).

Although more secondary, we will also question some possible differences in the framing effect function of the class of the dilemma, that is, either the bystander or footbridge versions of the trolley dilemma. This questioning largely derives from the dual-process model of moral judgment (Greene, 2001, 2004, 2008), for which the generic version of the model proposes that both emotional factors and deliberative ones are activated at the time of making a moral judgment. In classic moral dilemmas, not taking the action (harm-rejection) is commonly consistent with deontic principles (i.e., whereby the morality of an action is assessed based upon a set of duties and rights). By contrast, taking the action (outcome-maximization) is commonly consistent with utilitarian principles (i.e., whereby the morality of an action is assessed based on its consequence). There is evidence that harm-rejection is supported by more emotional processes compared to outcome-maximization (and vice-versa for deliberate processes; but see Baron, 2017; Gürçay \& Baron, 2016; Kahane, 2015, for other interpretations). In parallel, in order to address the discrepancy in moral judgments to the bystander and the footbridge versions of the dilemma, neuroimaging studies have suggested that emotion may be more important in response to the footbridge scenario (for which a greater activation of the medial prefrontal cortex, the posterior cingulate cortex, and the amygdala is observed) than to the bystander 
scenario (for which it is observed a greater activation of the dorsolateral prefrontal cortex) (Greene et al., 2001, 2004). It is worth noting that the two scenarios differ in many more aspects than just emotion (see Bauman et al., 2014). Despite some methodological limits inherent to the variations of the Trolley dilemma, these scenarios however, remain ones of the most studied, and allow to mirror the materiel used in the four studies we reported on framing effects in moral dilemmas.

Mixing the predictions of the dual-process theories of moral decision-making and framing susceptibility makes it possible to make specific predictions regarding the reliability of these judgments. Situations eliciting a stronger emotional response should be more affected by framing effects compared to situations eliciting a weaker emotional response. Applying this rationale to the trolley dilemma, one would expect that people would be more sensitive to the framing effect in the footbridge version than in the bystander version (i.e., interaction effect).

\section{Method}

\section{Participants}

Participants were recruited online via Amazon's Mechanical Mturk (mean age $=35$ years old; \% female $=46 \%$ female) and received financial compensation in accordance with the recommended minimum hour wage of 8-10\$ (Gleibs, 2017; Goodman \& Paolacci, 2017). To be part of the study, participants had to be between 18 and 50 years old, be native English speakers, reside in the United States and never have received a psychiatric diagnosis. A total of 1748 participants were randomly assigned to one condition of a 2 (scenario: footbridge vs. bystander) x 2 (framing: positive vs. negative) x 2 (framed outcome: 5 people vs. 1 people) between-subjects design. Of these, 31 did not provide any response and were thus not considered in statistical analyses. In addition, 663 participants were excluded from main analyses because they failed to correctly 
answer an attentional check (see Procedure section below). A total of 1054 participants with no missing value and with a correct attentional check could thus be included in final statistical analyses. However, because we registered our analyses for 130 participants per group, only the 130 first participants of each group were considered (i.e. a final total sample size of 1040 participants). Note that we report two sensitivity analyses in the Results section including either all participants with no missing value or all participants with a correct attentional check. A sequential stopping rule was planned but no additional participants were recruited as the effect on which the sequential stopping rule relied had a higher $p$-value than the cut-off used. The university ethics review board approved this research. Participants gave their written consent prior to participating in the study, in accordance with the Declaration of Helsinki.

\section{Materials and procedure}

Participants completed a framing problem based upon the trolley dilemma (Greene et al., 2001). After reading the scenario, participants were instructed to indicate whether taking the action was morally acceptable (i.e., pushing the man in the footbridge scenario, or pushing the lever in the bystander scenario) or not by using a visual analogic scale ranging from 1 "Not at all acceptable" to 7 "Totally acceptable". In classic trolley dilemmas, taking an action triggers two parallel outcomes at the same time (a first one concerning the group of 5 people and a second one concerning the 1 person). For instance, pushing the man off the bridge in the footbridge dilemma affects the survival of both the single man and the group of 5 people working on the railroad tracks. In classic goal framing effect paradigms, by contrast, participants are typically presented with a behavior affecting only one outcome (Levin et al., 1998). For example, in the classic study of Meyerowitz, performing a self-breast examination affects only one outcome - the probability to detect the cancer at an early stage (Meyerowitz \& Chaiken, 1987). With the purpose to align 
with the standard classification of goal framing effects without making any major changes to the initial trolley dilemma, we decided to manipulate the frame of one outcome only at a time (5 people or 1 person) while keeping the second outcome constant (see Table 1). When not subject to framing manipulation, the second outcome was described to the participants in a neutral formulation to ensure understanding of the dilemma. The split of these conditions not only served as a methodological precaution, but it was also used to conduct a replication of the influence of the goal framing over two conditions for each scenario, potentially strengthening the robustness of our conclusions. 
Table 1. Description of framing manipulation in the bystander and footbridge scenarios.

\section{Trolley dilemma}

\section{Footbridge scenario}

\section{Bystander scenario}

Outcome 1

Outcome 2

Outcome 1

Outcome 2

You are standing on a footbridge over a track, near to a man wearing a large, heavy backpack (which he can't take off). Under the bridge, five railway workmen are standing. Further on, you see a trolley hurtling down the same track.

In the event that the trolley pursues on its present course without being stopped (for example by having something heavy thrown onto the track), the 5 workers will be killed in the accident.

Positive If you don't push

Frame the man over the bridge, he will be the only one to survive.

Negative If you push the man Frame over the bridge, he will be the only one to die.
You are standing on a footbridge over a track, near to a man wearing a large, heavy backpack (which he can't take off). Under the bridge, five railway workmen are standing. Further on, you see a trolley hurtling down the same track.

In the event that the man falls on the track, he will be killed in the accident but his large heavy backpack will instantly stop the trolley
You are near to the switch of a railroad track. On the main track, there is a group of five railway workmen. On a side track, there is a single railway workman. Further on, you see a trolley hurtling down the main track.

In the event that the trolley pursues on its present course without being redirected, the 5 workers will be killed in the accident.
You are near to the switch of a railroad track. On the main track, there is a group of five railway workmen. On a side track, there is a single railway workman. Further on, you see a trolley hurtling down the main track.

In the event that the trolley is redirected to the side track, the single railway worker will be killed in the accident.
If you push the man If you don't push the over the bridge, the 5 switch, the single workers will be the railway workman only ones to survive. will be the only one to survive.

If you don't push the man over the bridge, the 5 workers will be the only ones to die.
If you push the switch, the single railway workers will be the only one to die.
If you push the switch, the 5 workers will be the only ones to survive.

If you don't push the switch, the 5 workers will be the only ones to die. 
An attention check was included, to ensure that participants allocate adequate attention to the task (e.g., Oppenheimer et al., 2009). Participants in the 'active' description condition (i.e., in which the outcome of pushing the man or pulling the lever is framed) were asked to describe the outcome of the counterfactual, passive decision (e.g., Should you have not pushed the man onto the track, what would have happened for the one man, and for the group of five men?). Similarly, participants in the 'passive' condition (i.e., in which the outcome of not pushing the man or not pushing the lever is framed) were asked to describe the outcome of performing the action. As previously described, participants who failed to report the accurate outcomes were excluded from the final analyses.

\section{Planned statistical analysis}

R code and exhaustive results of the statistical analyses are available in Supplementary Materials. The effect of scenario on framing susceptibility was assessed by conducting a 3-way independent measure analysis of variance (ANOVA) with Outcome (1 versus 5), Scenario (footbridge versus bystander) and Frame (positive vs. negative) as predictors, and moral acceptability (from 1 "Not at all acceptable" to 7 "Totally acceptable") as the dependent variable in $\mathrm{R}$ environment. A detailed description of the planned analyses can be found from OSF (https://osf.io/8r4sk/).

\section{Results}

\section{Registered analyses}

First, as a data quality check, we explored if our sample accepted sacrificial harm more in the 'bystander' than in the 'footbridge' scenario. We found a large effect of scenario in the hypothesized direction (see Table 1). This result thus affords some indication about the quality of the data collected. 
Second, we tested our main hypothesis by exploring if positive frame led to increased acceptance of sacrificial harm compared to negative frame. Unexpectedly, the main effect of frame was not statistically significant: overall, judgments did not differ between positive $(M \pm$ $\left.S D ; M_{\text {Acceptability }}=4.04 \pm 2.04\right)$ and negative frame $\left(M_{\text {Acceptability }}=4.00 \pm 2.15\right)$, associated with a very small effect size (partial $\eta^{2}<.001$; see Table 2). Equivalence tests revealed that this main effect of framing falls within the equivalence bound of Cohen's $d=-0.15$ and Cohen's $d=0.15$.

Table 2. Results of the 3-way ANOVA with moral acceptability as the dependent variable

\begin{tabular}{rccc}
\hline Predictor & F-test & p-value & ${\text { Partial } \boldsymbol{\eta}^{\mathbf{2}}}^{\text {Frame }}$ \\
Scenario & 0.12 & .725 & $<.001$ \\
Outcome & 181.35 & $<.001$ & .15 \\
Frame x Scenario & 1.17 & .280 & .001 \\
Frame x Outcome & 0.09 & .768 & $<.001$ \\
Scenario x Outcome & 3.96 & .047 & .004 \\
Frame x Scenario x Outcome & 0.07 & .785 & $<.001$ \\
\hline
\end{tabular}

Finally, we conducted an exploratory analysis investigating whether the magnitude of framing susceptibility differed in each scenario. Again, we found no evidence supporting our hypothesis since neither the Frame x Scenario interaction nor the Frame x Scenario x Outcome interaction reached statistical significance; and the associated effect sizes were very small $\left(\eta^{2}<\right.$ $.001)$.

\section{Additional analyses}

A more exhaustive presentation of the results of these additional analyses can be found in Supplementary Materials S2, and S3. Although unanticipated, our results revealed a statistically significant Frame x Outcome interaction $\left(F(1,1032)=3.96, p=.047, \eta_{p}{ }^{2}=.004\right)$. As shown in 
Figure 1, participants in the 'one person condition' tended to exhibit a standard framing effect (i.e., higher moral acceptance of sacrificial harm in the positive versus negative frame) while participants in the 'five persons' condition tended to exhibit a reversed framing effect (i.e., decreased acceptance of sacrificial harm in the positive versus negative frame). Notably, all the associated effect sizes were very small. Our sample size (260 participants in each condition) was not sufficient to detect statistically significant effects (framing effect in the 'one person' condition: Cohen's $\mathrm{d}=--0.15, p>.20$; framing effect in the 'five persons' condition: Cohen's $\mathrm{d}$ $=0.10, p=.49 ;$ see Supplementary Materials S5). Equivalence tests revealed that effect sizes fall within the equivalence bound of Cohen's $d=-0.35$ and Cohen's $d=0.35$ in the two Outcome conditions.

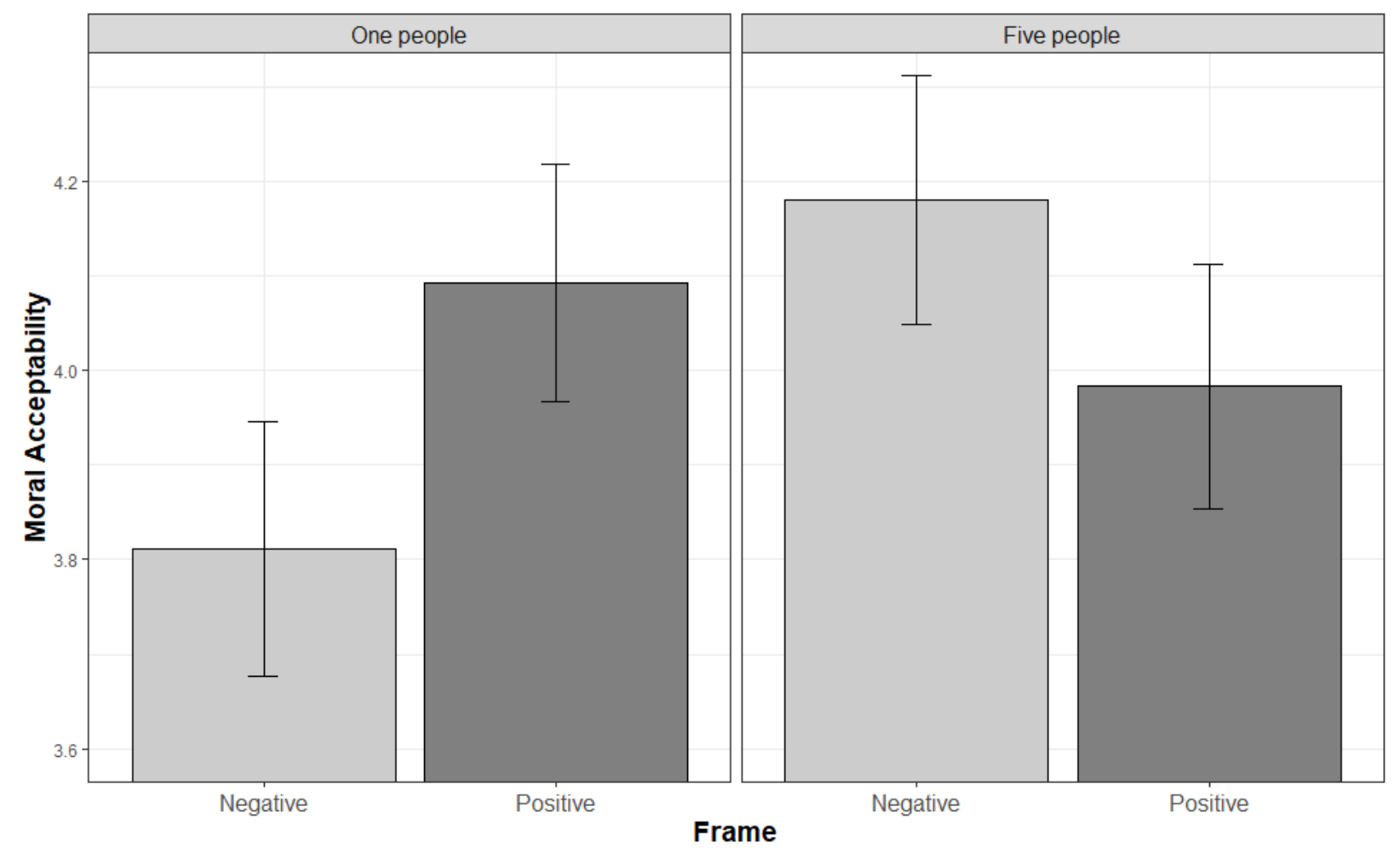

Figure 1. Framing susceptibility in the two Outcome conditions. 
Then, diagnostics of the 3-way ANOVA revealed that the variance was not homogeneous across conditions and that residuals were not normally distributed (although they were distributed symmetrically around 0; see Supplementary Materials S2). Although the Central Limit Theorem may be invoked given our large sample size, these issues were nevertheless investigated in three successive analyses to assess the robustness of our findings. First, we identified influential points using Cook's distance and re-ran the ANOVA excluding participants with a high Cook's distance (we relied on the commonly used 4/sample size value as cut-off; see Gogoi, 2015). Removing the 22 identified participants did not improve the variance homogeneity or the distribution of the residuals. Second, because our dependent variable had a bimodal distribution, we transformed it using a Box-Cox transformation. Several values of lambda were used. The 3-way ANOVA was re-run using the transformed values as dependent variables. Again, the variance remained not homogeneous across conditions and the distribution of the residuals did not achieve normality. As a last analysis, we ran a robust ANOVA which does not make any assumption about normality or variance homogeneity. The main effects of scenario and framing remained respectively significant and non-significant in these 5 re-analyses of our primary model. Only the Frame x Outcome interaction saw its $p$-value increase following the robust ANOVA, which became marginally significant ( $p=0.070$, see Supplementary Materials S2).

Finally, as previously described in the method section, a substantial proportion of our initial sample was not retained in the present analyses; the reasons are twofold. First, we excluded all the participants who failed the attention check. Second, due to the randomization system used by the platform employed to collect data, some experimental conditions were filled with a number of participants slightly higher than expected. Since our analyses were registered 
with 130 participants per condition, we selected the 130 first participants in each condition to perform the analyses reported in the paper. In two distinct sensitivity analyses, we reproduced our main model including this time (i) all participants (regardless of their response to the attention check) or (ii) only participants with a correct attentional check. Including all the participants who successfully answered the attention check did not affect the results (see Supplementary Materials S3). By contrast, including all participants (regardless of their response to the attention check), made the Frame x Outcome interaction become statistically nonsignificant (all other effects remained unchanged).

\section{Discussion}

We conducted a pre-registered conceptual replication exploring whether decisions in a classic moral dilemma may be shaped by the framing effect. Our data revealed that, across scenarios and outcomes, the decisions of participants did not statistically differ between the two framing conditions. This result was thus contrary to what we have expected. However, a significant but unplanned interaction revealed that decisions of participants are affected differently by the framing depending on the outcome framed. When framing manipulation targeted only one person (the sole railway worker in the bystander scenario or the man over the bridge in the footbridge scenario), the positive frame tended to elicit more utilitarian-like judgments compared to the negative frame. This result is consistent with all the previous studies that have reported a framing effect in the trolley dilemmas: in all these studies, the positive frame always increased acceptance of sacrificial harm compared to the negative frame (Broeders et al., 2011; Cao et al., 2017; Petrinovich et al., 1996). However, when the framing manipulation targeted the group of five railway workers, the positive frame tended to elicit lower acceptance of sacrificial harm compared to the negative frame. We nevertheless believe that caution is warranted addressing 
this unexpected result, at least for three main reasons. First, from a methodological perspective, our material was not specifically designed to explore this interaction. Future studies may target this phenomenon, using multiple scenarios and making the number of persons involved vary (e.g., Trémolière et al., 2014) so as to provide greater insight into the moderating role of the outcome framed in framing susceptibility. Second, the associated effect sizes are very low and our study was not powered to detect such low effect sizes. The magnitude of framing effect was around Cohen's $d=|0.10|$ in the 'one person or the 'five persons' conditions and thus did not reach statistical significance (around 2000 participants per group are required to achieve 95\% power at an alpha of .05 to detect this effect size). Third, this result was not robust to sensitivity analyses. The use of robust ANOVA made the $p$-value of this interaction become only marginally significant. In the same way, including all the participants regardless of their response to the attentional check, made the $p$-value of this interaction become not significant. More robust evidence is thus required before drawing firm conclusions about the moderator effect of outcome on framing susceptibility in the trolley dilemmas.

Overall, we observed a considerably weaker framing effect than expected and traditionally observed in the decision making literature. For instance, a 2005 comprehensive meta-analysis of classic goal framing studies revealed a mean effect size about $d=0.44,95 \% \mathrm{CI}$ $=[0.36,0.53]$ (Piñon \& Gambara, 2005). In our study, the equivalence tests allowed us to conclude that the effect size of our main effect of framing was significantly lower than Cohen's d $=|0.15|$. Because the interaction between the frame and the outcome reached statistical significance, we also re-ran equivalence tests in each of the two outcome modalities. Despite the lower sample size, equivalence tests allowed us to conclude that the effect size of the framing effect was significantly lower than Cohen's $d=|0.35|$. Two main explanations, we believe, may 
explain this result. First, participants showed a particularly weak framing effect in our study, possibly because moral decisions in the context of sacrificial dilemmas are less susceptible to framing manipulation - and are therefore more reliable - than decisions made in some other situations. This explanation is supported by the work of Helzer et al. (2017), which showed consistency in utilitarian judgments in the context of sacrificial dilemmas across multiple contexts and over time. An alternative explanation relies on the finding in the framing literature, that low stake decisions produce weaker framing effects compared to high stake decisions (Gosling et al., 2020; Seta et al., 2017). Numerous authors in the moral judgment field have pointed the lack of realism of commonly used sacrificial dilemmas (e.g., Bauman et al., 2014). Although we acknowledge it is speculative, the weak effect sizes found in the present study may result from the low importance attributed by the participants to the fictitious scenarios; what may, in turn, enhance their ability to resist framing manipulation. Future studies investigating framing susceptibility in dilemmas with either a low or high level of realism (e.g., Körner et al., 2019) might increase our understanding of the influence of framing in sacrificial moral dilemmas.

Last, previous literature on this topic shows hardly reconcilable results (some studies observing framing effects in the 'foodbridge' but not in the 'bystander' scenario, e.g., Broeders et al., 2011, while other showing the opposing pattern of results, e.g., Cao et al., 2017). Our preregistered, well-powered study showed no overall effect of framing on acceptance of sacrificial harm no matter what the scenario. Equivalence tests confirmed that the effect sizes associated with framing were all very low. The differences in the decision tasks used in the studies applying framing effects to morality may also deserve attention in the attempts to explain the inconsistent patterns of results present in the literature. For instance, some studies give 
participants the role of actors who are given the possibility to "take an action" (e.g., Cao et al., 2017) when other studies, just like ours, rather give the participants the role of observing judges (e.g., Pastotter et al., 2011). Acknowledging the existing differences between having participants take the role of observers or actors in moral judgment situations (Gold et al., 2015), the design of the decision task may also be worth investigating. Given our findings, we call for future wellpowered, direct and conceptual replications to be conducted to provide a clearer picture of the role of framing effects in the trolley dilemma and more.

Countless studies have emphasized the framing effect in the decision-making field, testifying to its robustness in this field (Kühberger et al., 1999; Steiger \& Kühberger, 2018). Although we can only deplore the weak number of studies applying the framing manipulation in standard sacrificial dilemmas, the handful of studies conducted on the topic show less convincing results. Replications of these studies as well as direct comparisons of framing susceptibility in morally-charged versus non-morally charged situations will provide valuable insights into people's ability to resist framing manipulation when moral decision are at stake. 


\section{References}

Allman, J., \& Woodward, J. (2008). What are moral intuitions and why should we care about them? A neurobiological perspective. Philosophical Issues, 18, 164-185.

Baron, J. (2017). Utilitarian vs. deontological reasoning: method, results, and theory. In J. F. Bonnefon \& B. Trémolière (Eds.), Moral inferences (pp.137-151). Hove, UK: Psychology Press

Bauman, C. W., McGraw, A. P., Bartels, D. M., \& Warren, C. (2014). Revisiting external validity: Concerns about trolley problems and other sacrificial dilemmas in moral psychology. Social and Personality Psychology Compass, 8(9), 536-554.

Bilandzic, H., Kalch, A., \& Soentgen, J. (2017). Effects of goal framing and emotions on perceived threat and willingness to sacrifice for climate change. Science Communication, 39(4), 466-491.

Broeders, R., Van Den Bos, K., Müller, P. A., \& Ham, J. (2011). Should I save or should I not kill? How people solve moral dilemmas depends on which rule is most accessible. Journal of Experimental Social Psychology, 47(5), 923-934.

Cao, F., Zhang, J., Song, L., Wang, S., Miao, D., \& Peng, J. (2017). Framing effect in the trolley problem and footbridge dilemma: Number of saved lives matters. Psychological reports, 120(1), 88-101.

Casey, L. S., Chandler, J., Levine, A. S., Proctor, A., \& Strolovitch, D. Z. (2017). Intertemporal differences among MTurk workers: Time-based sample variations and implications for online data collection. SAGE Open, 7(2), 1-15. 
Christensen, J. F., \& Gomila, A. (2012). Moral dilemmas in cognitive neuroscience of moral decision-making: A principled review. Neuroscience \& Biobehavioral Reviews, 36(4), $1249-1264$.

De Martino, B., Kumaran, D., Seymour, B., \& Dolan, R. J. (2006). Frames, biases, and rational decision-making in the human brain. Science, 313, 684-687.

Demaree-Cotton (2016) Do framing effects make moral intuitions unreliable?, Philosophical Psychology, 29, 1-22,

Epstein, S. (1994). Integration of the cognitive and psychodynamic unconscious. American Psychologist, 49, 709-724.

Evans, J. S. (2008). Dual-processing accounts of reasoning, judgment and social cognition. Annual Review of Psychology, 59, 255-278.

Evans, J. S. B. T., \& Over, D. E. (1996). Rationality and reasoning. Hove, UK: Psychology Press.

Foot, P. (1978). The problem of abortion and the doctrine of double effect. In: Virtues and Vices. Oxford: Blackwell.

Gleibs, I. H. (2017). Are all "research fields" equal? Rethinking practice for the use of data from crowdsourcing market places. Behavior Research Methods, 49(4), 1333-1342.

Gogoi, B. (2015). Influential Observations and Cutoffs of Different Influence Measures in Multiple Linear Regression. International Journal of Computational and Theoretical Statistics, 2(02). 
Gold, N., Pulford, B. D., \& Colman, A. M. (2015). Do as I say, don't do as I do: Differences in moral judgments do not translate into differences in decisions in real-life trolley problems. Journal of Economic Psychology, 47, 50-61.

Goodman, J. K., \& Paolacci, G. (2017). Crowdsourcing consumer research. Journal of Consumer Research, 44(1), 196-210.

Gosling, C. J., Caparos, S., \& Moutier, S. (2020). The interplay between the importance of a decision and emotion in decision-making. Cognition and Emotion. Doi : $10.1080 / 02699931.2020 .1741340$

Greene, J. D., Morelli, S. A., Lowenberg, K., Nystrom, L. E., \& Cohen, J. D. (2008). Cognitive load selectively interferes with utilitarian moral judgment. Cognition, 107, 1144-1154.

Greene, J. D., Nystrom, L. E., Engell, A. D., Darley, J. M., \& Cohen, J. D. (2004). The neural bases of cognitive conflict and control in moral judgment. Neuron, 44, 389-400.

Greene, J. D., Sommerville, R. B., Nystrom, L. E., Darley, J. M., \& Cohen, J. D. (2001). An fmri investigation of emotional engagement in moral judgment. Science, 293, 2105-2108.

Gürçay, B., \& Baron, J. (2016). Challenges for the sequential two-system model of moral judgement. Thinking \& Reasoning, 23, 49-80.

Haidt, J. (2001). The emotional dog and its rational tail. Psychological Review, 108, 814-834.

Helzer, E. G., Fleeson, W., Furr, R. M., Meindl, P., \& Barranti, M. (2017). Once a utilitarian, consistently a utilitarian? Examining principledness in moral judgment via the robustness of individual differences. Journal of personality, 85(4), 505-517. 
Kamm, F., 1993. Morality, Mortality, Volume I: Death and Whom to Save From It. Oxford University Press, New York.

Kahane, G. (2015). Sidetracked by trolleys: Why sacrificial moral dilemmas tell us little (or nothing) about utilitarian judgment. Social Neuroscience, 10, 551-560.

Kahneman, D. (2011). Thinking, fast and slow (Vol. 1). New York: Farrar, Straus and Giroux.

Kahneman, D., \& Frederick, S. (2007). Frames and brains: Elicitation and control of response tendencies. Trends in Cognitive Sciences, 11, 45-46.

Kim, J., Kim, J. E., \& Marshall, R. (2014). Search for the underlying mechanism of framing effects in multi-alternative and multi-attribute decision situations. Journal of Business Research, 67(3), 378-385.

Kohlberg, L. (1969). Stage and sequence: The cognitive-developmental approach to socialization. In D. A. Goslin (Ed.), Handbook of socialization theory and research (p. 347-480). Chicago: Rand

Körner, A., Joffe, S., \& Deutsch, R. (2019). When skeptical, stick with the norm: Low dilemma plausibility increases deontological moral judgments. Journal of Experimental Social Psychology, 84, 103834.

Kühberger, A., Schulte-Mecklenbeck, M., \& Perner, J. (1999). The effects of framing, reflection, probability, and payoff on risk preference in choice tasks. Organizational Behavior and Human Decision Processes, 78, 204-231.

Lakens, D. (2017). Equivalence tests: a practical primer for t tests, correlations, and metaanalyses. Social Psychological and Personality Science, 8(4), 355-362 
Levin, I. P., \& Gaeth, G. J. (1988). How consumers are affected by the framing of attribute information before and after consuming the product. Journal of consumer research, 15(3), 374-378.

Levin, I., Schneider, S., \& Gaeth, G. (1998). All frames are not created equal: A typology and critical analysis of framing effects. Organizational Behavior and Human Decision Processes, 76, 149-188.

McGuire, J., Langdon, R., Coltheart, M., \& Mackenzie, C. (2009). A reanalysis of the personal/impersonal distinction in moral psychology research. Journal of Experimental Social Psychology, 45(3), 581-584.

Meyerowitz, B. E., \& Chaiken, S. (1987). The effect of message framing on breast selfexamination attitudes, intentions, and behavior. Journal of personality and social psychology, 52(3), 500.

Murch, K. B., \& Krawczyk, D. C. (2013). A neuroimaging investigation of attribute framing and individual differences. Social cognitive and affective neuroscience, 9(10), 1464-1471.

Oppenheimer, D. M., Meyvis, T., \& Davidenko, N. (2009). Instructional manipulation checks: Detecting satisficing to increase statistical power. Journal of Experimental Social Psychology, 45(4), 867-872.

Pastötter, B., Gleixner, S., Neuhauser, T., \& Bäuml, K. H. T. (2013). To push or not to push? Affective influences on moral judgment depend on decision frame. Cognition, 126(3), $373-377$ 
Petrinovich, L., \& O’Neill, P. (1996). Influence of wording and framing effects on moral intuitions. Ethology and Sociobiology, 17, 145-171.

Piñon, A., \& Gambara, H. (2005). A meta-analytic review of framming effect: risky, attribute and goal framing. Psicothema, 17(2), 325-331

Seta, J. J., Seta, C. E., \& McCormick, M. (2017). Commonalities and differences among frames: a unification model. Journal of Behavioral Decision Making, 30(5), 1113-1130.

Sinnott-Armstrong, W. (2011). Emotion and reliability in moral psychology. Emotion Review, 3, 288-289.

Sinnott-Armstrong, W. (Ed.). (2008). Moral psychology. Cambridge, MA: MIT Press.

Sloman, S. A. (1996). The empirical case for two systems of reasoning. Psychological Bulletin, 119, 3-22.

Stanovich, K. E. (1999). Who is rational? Studies of individual differences in reasoning. Mahwah, NJ: Lawrence Erlbaum.

Steiger, A., \& Kühberger, A. (2018). A meta-analytic re-appraisal of the framing effect. Zeitschrift für Psychologie, 226(1), 45.

Thomson J (1985) The Trolley Problem. Yale Law Journal, 94, 1395-1415.

Tversky, A., \& Kahneman, D. (1981). The framing of decisions and the psychology of choice. Science, 211, 453-458. 
MORAL DECISION-MAKING AND FRAMING EFFECT 